\title{
Entre los tiempos escolares y los tiempos de ocio: Su incidencia en la vida cotidiana de los adolescentes que cursan la Educación Secundaria obligatoria en España ${ }^{1}$
}

\section{Between the school times and the times of leisure: its incidence in the daily life of the teenagers who attend the Secondary obligatory Education in Spain}

\author{
$\mathrm{M}^{\mathrm{a}}$ Belén Caballo ${ }^{2}$ \\ José Antonio Caride ${ }^{3}$ \\ Rita Gradaílle
}

\begin{abstract}
RESUMEN
El artículo se inscribe a una línea de investigación a la que se vinculan diferentes contribuciones realizadas en los últimos años por el Grupo de Investigación de Pedagogía Social y Educación Ambiental (SEPA) de la Universidad de Santiago de Compostela-España; financiados en las Convocatorias de Ayudas a Proyectos de Investigación del Plan Nacional de
\end{abstract}

1 El texto que se presenta toma como referencia el proyecto de investigación "Tiempos escolares y tiempos de ocio: problemáticas especificas y alternativas pedagógico-sociales en la vida cotidiana del alumnado de Educación Secundaria Obligatoria", financiado con Fondos FEDER de la Unión Europea, a través de convocatoria pública del Ministerio de Ciencia e Innovación de España (Plan Nacional de $I+D+i$ 2008-2011), siendo su Investigador Principal el Prof. Dr. José Antonio Caride.

2 Doutora en Ciencias de la Educación. Profesora Doutora del Departamento de Teoría e Historia de la Educación y Pedagogía Social de la Universidad de Santiago de Compostela, España.

3 Doutor en Ciencias de la Educación. Profesor Catedrático del Departamento de Teoría e Historia de la Educación y Pedagogía Social de la Universidad de Santiago de Compostela, España.

4 Doutora en Ciencias de la Educación. Profesora Doutora del Departamento de Teoría e Historia de la Educación y Pedagogía Social de la Universidad de Santiago de Compostela, España. 
I $+\mathrm{D}+\mathrm{i}$ que, genéricamente, toman como referencia los tiempos educativos y los tiempos de ocio en los procesos de socialización y la vida cotidiana de la infancia-adolescentes en la sociedad red. Con esta perspectiva, las contribuciones específicas del artículo inciden en los significados -viejos y nuevos- que los tiempos educativos, y muy especialmente los relacionados con el calendario y horarios escolares, tienen en la vida cotidiana de los adolescentes (12-16 años) que cursan la Educación Secundaria Obligatoria (ESO) en España. En este sentido, se valoran algunos de los problemas emergentes en la sociedad red, así como las alternativas pedagógico-sociales que deberán adoptarse para un desarrollo más integral de su formación y de los derechos que se invocan en nombre del ocio y de su educación.

Palabras clave: tiempos escolares; tiempos de ocio; educación secundaria; adolescencia; vida cotidiana.

\begin{abstract}
The article relates to a line of investigation linked to the various contributions made in the last years by the Social Pedagogy and Environmental Education research group (SEPA) of the University of Santiago of Compostela-Spain; financed in the Announcements of Helps to Projects of Investigation of the National Plan of $\mathrm{I}+\mathrm{D}+\mathrm{i}$ that, generically, taking as a reference the educational times and the times of leisure in the processes of socialization and the daily life of the infancy-teenagers in the network society. With this perspective, the specific contributions of the article influence the meanings -old and new- education times, and very especially the related with the calendar and school hours, have in the daily life of the teenagers (12-16 years) that study the Secondary Obligatory Education in Spain. In this sense, are valued some problems emerging in the network society, as well as the social pedagogic alternatives to be adopted for development more comprehensive training and rights that are invoked on behalf of leisure and education.
\end{abstract}

Keywords: school times; times of leisure; secondary education; adolescence; daily life.

\title{
Introducción: cambiar los tiempos de la educación
}

El reconocimiento explícito de que podemos educar y educarnos a lo largo de toda la vida (DELORS, 1996), poniendo en valor los múltiples escenarios que habilitan los procesos formativos -ya sea en las familias o en las escuelas, 
en las comunidades locales o en las redes virtuales-, cuestiona radicalmente las nociones temporales que han marcado el quehacer educativo durante siglos; y, con ellas, una concepción de la enseñanza y los aprendizajes esencialmente monocrónica y burocrática (HARGREAVES, 1992), en la que el tiempo se nos muestra como un potente mecanismo disciplinario (FOUCAULT, 1982), regulador del control institucional que determinados poderes ejercen sobre los esquemas pedagógicos y curriculares de los centros escolares, sobre las relaciones establecidas entre el alumnado y el profesorado, los métodos y los saberes (COMPÈRE, 1997). También, e inevitablemente, sobre los modos de socializarse en los códigos morales y culturales de un modelo de sociedad, al que los sistemas escolares debían sustentar y reproducir (ESCOLANO, 2000).

Iniciándose el tercer milenio, en la sociedad de la información y del conocimiento, los tiempos de y/o para la educación ya no son lo que eran, ni en sus construcciones teóricas ni en sus consecuencias prácticas. No deberían serlo; menos aún si se insiste en observar el tiempo como un simple recurso organizativo, cuantificable y lineal, al que es preciso adecuar el ordenamiento estructural de los sistemas educativos (atendiendo a edades, ciclos, niveles, cursos, etc.), la programación secuenciada de los contenidos curriculares, la apertura y cierre de los centros escolares, o la conciliación de los calendarios y horarios lectivos con otros ritmos sociales. Siendo importantes y necesarios para seguir alentando -y, en muchos casos, garantizando- una educación para todos, no bastan. $\mathrm{Ni}$ sirven para sostener la escuela, cuyos modelos de planificación y distribución del tiempo siguen pautándose rindiendo tributo a los convencionalismos heredados, sin que apenas hayan penetrado en ellos "los cambios producidos en los principios pedagógicos, biológicos, psicológicos y sociales de la enseñanza" (HUSTI, 1992, p. 278); ni sirven para construir la educación del mañana que necesitamos, en un mundo cargado de incertidumbres, complejo y globalizado, que ha de comenzar por transformar la educación de hoy (CARBONELL, 2008, p. 21): una "educación de tiempos lentos, tranquilos y prolongados", que respete los ritmos naturales de la infancia y la adolescencia, que sea flexible en los modos de programar las enseñanzas y los aprendizajes, de transitar de lo escolar a lo extraescolar y al ocio... Un tiempo que no sea el enemigo de la libertad, ni de los profesores ni de los alumnos, "sino su compañero de camino y su apoyo" (HARGREAVES, 1992, p. 53).

Insistimos en ello cuando todo indica que "la red" ha cambiado decisivamente nuestras percepciones acerca del tiempo, para vivirlo y apreciarlo con otros parámetros, por muy controvertidos y paradójicos que sean (CEBRIÁN, 1988; CASTELLS, 1997): un tiempo de tiempos, atemporal y apresurado, en el que la celeridad de los acontecimientos socava la idea misma del tiempo. El síndrome de la prisa, que abona la pasión por el presente, tiende a borrar cualquier pasado 
y a desconfiar de todo futuro, por lo que resulta imprescindible resignificar su sentido en nuestras sociedades. Hacerlo para que sea un "tiempo oportuno" en el que confluyan "dos dimensiones temporales que hoy aparecen dramáticamente separadas y enfrentadas: el tiempo de la vida y el tiempo del mundo, o, si se prefiere, el tiempo privado y el tiempo público" (MARRAMAO, 2008, p. 17). Un antagonismo que la educación escolar ha contribuido a agrandar y perpetuar.

No obstante, sean cuales sean sus circunstancias, el tiempo siempre tendrá valor y resultará valioso para la educación (GIMENO, 2007). No sólo porque se trata de un bien escaso y limitado que afecta a todo lo que somos y hacemos cotidianamente (DURÁN, 2007); sino también porque las lecturas que se hagan acerca del tiempo ya no pueden obviar la necesidad de interpretar y experimentar sus realidades con otros significados, pedagógica y socialmente alternativos a los ya conocidos, en una sociedad abierta las 24 horas. Entre otras opciones, la pedagogía-educación del ocio (CUENCA, 2004; ELIZALDE, 2010) y las reformas que se emprendan en los sistemas educativos, a nivel nacional e internacional, deben asumir con todas sus consecuencias las oportunidades que este cambio de paradigma requiere, tomando en consideración la riqueza teórico-conceptual, metodológica y empírica que los estudios del tiempo han adquirido en la investigación educativa y, por extensión, en la investigación social en las últimas décadas (véanse, entre otros: RAMOS, 1992; ROMERO, 2000; PEREYRA, 2005; TABBONI, 2006; GIMENO, 2007; DURÁN; ROGERO, 2010; CABALLO; CARIDE, 2011; CAVET, 2011).

\section{Los tiempos educativos de los adolescentes escolarizados en la ESO: una aproximación al conocimiento de sus realidades}

En estas coordenadas científicas y académicas, como un proyecto de investigación asociado a una línea de trabajo a la que se adscriben diferentes contribuciones realizadas por el Grupo de Investigación en "Pedagogía Social y Educación Ambiental" (SEPA), de la Universidad de Santiago de Compostela desde los años noventa del pasado siglo, situamos nuestra intención de conocer los significados -viejos y nuevos- que los tiempos educativos, y muy especialmente los relacionados con el calendario y horario escolar, tienen en la vida cotidiana de los adolescentes escolarizados en la Educación Secundaria Obligatoria (ESO) en España, de sus problemas emergentes y de las alternativas que han de adoptarse para el desarrollo integral de su personalidad y de sus derechos cívicos. A lo que se añade una especial preocupación por los espacios y tiempos de ocio, cada vez más significativos para los aprendizajes y experiencias que tienen como 
soporte los vínculos sociales que en ellos se generan. Con esta perspectiva, los objetivos que se pretendían alcanzar se resumen, básicamente, en:

1. Identificar y analizar la distribución de los ritmos temporales que adoptan las instituciones educativas en distintos contextos sociales, valorando su incidencia en la vida cotidiana (procesos de socialización, relaciones familiares, etc.) de los adolescentes escolarizados.

2. Analizar las necesidades y expectativas de los adolescentes en relación con sus tiempos libres, tomando en consideración los condicionantes que imponen los calendarios y horarios.

3. Estudiar las prácticas culturales, recreativas, etc. en las que participan los adolescentes escolarizados en su tiempo libre, y su consideración como una experiencia de ocio, identificando propuestas y experiencias que posibiliten actuaciones alternativas (buenas prácticas) en la Educación del Ocio de los y las adolescentes.

Con un planteamiento metodológico plural y diversificado en los modos de conocer e interpretar, la triangulación de métodos (cuantitativos y cualitativos), técnicas y fuentes, constituyó uno de los ejes principales del estudio realizado, con una doble perspectiva: de un lado, posibilitar que los diferentes actores pedagógicos (profesorado, alumnado, familias, equipos directivos, etc.) tomasen la palabra, dando cauce a sus valoraciones e interpretaciones "subjetivas"; de otro, indagar de forma extensiva y, si cabe "representativa" las problemáticas y alternativas inherentes a la transición de los tiempos escolares a los tiempos sociales (con especial énfasis en los tiempos de ocio). En todo caso, siendo dos enfoques que se integran asumiendo un enfoque metodológico que permitiese la adaptación concreta de las estrategias y procedimientos de investigación a los sujetos y a las realidades objeto de estudio. A este planteamiento se asoció un trabajo de campo cuya realización tuvo lugar durante los cursos académicos 2009-10 y 2010-11:

- Cuestionarios elaborados ad hoc, a cumplimentar por alumnos, profesores, equipos directivos y familiares (padres y/o madres de alumnos) de centros que imparten la ESO en las 17 Comunidades Autónomas de la geografía española, así como por empresas-instituciones que prestan "servicios de ocio".

- Estudio de casos e identificación de buenas prácticas, tanto en la gestión del tiempo escolar, como en iniciativas (programas, experiencias, etc.) de Educación del Ocio.

- Grupos de discusión (entrevistas colectivas) con distintos sectores y agentes de la comunidad educativa, fundamentalmente con adolescentes escolarizados en la ESO. 
- Análisis de dietarios, semanarios o agendas personales de adolescentes escolarizados en la ESO.

Sin que podamos extendernos en una explicación exhaustiva de los procesos muestrales y de las razones que motivan sus respectivos criterios de validez y fiabilidad, diremos que el número de cuestionarios aplicados al alumnado se concretó en un $\mathrm{N}=3306$ (nivel de confianza del 99\%, con un error muestral del 2,24; $\mathrm{p}=\mathrm{q}=50$ ), siendo su distribución equitativa en función del género y de los cuatro cursos académicos de la ESO. Un $89 \%$ del total tiene edades comprendidas entre los 12 y los 15 años; el 82,6\% viven en zonas urbanas y el 17,4\% en medios rurales, mostrando un elevado grado de concordancia con la distribución de la Educación Secundaria en España; además, un 50,8\% cursaba la ESO en centros públicos, un $44,1 \%$ en centros privados concertados (financiados por el Estado) y un $5,0 \%$ en privados. Con el mismo perfil de los centros y de los contextos sociogeográficos en los que fueron aplicados los cuestionarios al alumnado, la muestra de profesores conforma un $\mathrm{N}=480$, con niveles de confianza del $95 \%$ y un error muestral del 4,$5 ; \mathrm{p}=\mathrm{q}=50$ ).

En el sistema educativo español, la escolaridad obligatoria se concibe como un servicio público, que el Estado tiene el deber de proporcionar atendiendo a los dos grandes niveles que conforman la educación básica: la Educación Primaria (6-12 años) y la Educación Secundaria Obligatoria (12-16 años), ambas gratuitas para todos los alumnos. Salvo que medien decisiones de la Administración Educativa autonómica o municipal, las familias han de costear los gastos correspondientes al material escolar y a los libros de texto, así como el uso de determinados servicios complementarios de comedor y transporte, para los que suelen habilitarse ayudas destinadas a las familias con menos recursos económicos.

La Educación Secundaria Obligatoria (ESO), se imparte en centros de educación secundaria, de titularidad pública (denominados Institutos de Educación Secundaria, IES) o privada, que pueden ofrecer además de estas enseñanzas, las de Bachillerato y formación profesional, de carácter postobligatorio. En general, los centros que ofertan la ESO deben impartir los cuatro cursos que la componen, con al menos una unidad por cada curso, y disponer de instalaciones como: aula-taller, laboratorio de ciencias experimentales, biblioteca, gimnasio, patio de recreo, aula de música, informática y plástica, espacios destinados a la actividad docente, de dirección y gestión del centro, etc. La ley prevé un máximo de 30 alumnos por aula, de obligado cumplimiento en toda España, independientemente la titularidad del centro y de los fondos que los sostienen.

La Educación Secundaria Obligatoria (ESO), a la que se accede al finalizar la Educación Primaria, comprende cuatro años académicos, que se cursan -ordinariamente- entre los 12 y los 16 años de edad. No obstante, los alumnos pueden 
permanecer escolarizados hasta el curso académico en el que cumplan los 18 años de edad. Normalmente, los grupos-aula se forman en función del año de nacimiento, teniendo cada uno de ellos asignado un profesor-tutor, aún cuando cada materia o área de conocimiento se imparta por un/a profesor/a especialista. En el curso académico 2007/08, en el que se inició el trabajo de campo de la investigación que presentamos, cursaban la ESO un total de 1.829.874 alumnos (lo que supone el cien por cien de tasa neta de escolarización para las edades de referencia, 12-16 años), de los que un 66,1\% lo hacían en centros públicos... La media de alumnos por aula no llegaba a los 25 alumnos, siendo inferior en los centros de titularidad pública $(23,6)$ que en los de titularidad privada: 26,2. Las tasas brutas de graduación son inferiores al 70 por ciento, lo que supone un índice de fracaso o abandono escolar que afecta al 31,7\% de los alumnos matriculados, con un reparto desigual cuando se comparan mujeres $(71,6 \%$ de tasa de graduación) y hombres, sensiblemente inferior: el 62,8 por ciento.

El calendario escolar suele iniciarse a mediados del mes de septiembre, finalizando en torno a la segunda semana de junio, constando de un mínimo de 175 días lectivos -que fijan anualmente las Administraciones Educativas de cada Comunidad Autónoma-, repartidos en 35 semanas de cinco días lectivos. La distribución de las vacaciones supone que aproximadamente 12 semanas correspondan al periodo estival, 15 días a las Navidades y entre 7-10 días a la Semana Santa; a estos días se añaden los de otras festividades declaradas por las Administraciones Autonómicas o Locales, en distintos momentos del año. La semana escolar en la ESO transcurre de lunes a viernes, en las que se distribuyen un total de 30 sesiones lectivas de 55-60 minutos de duración cada una de ellas, lo que representa hasta 6 sesiones diarias; esto es: un número anual mínimo de 1.050 horas lectivas anual. Más allá de este horario, cada centro debe especificar las horas y condiciones en las que estará abierto, poniendo a disposición de la comunidad educativa, los servicios y equipamientos que se estime oportuno.

La distribución de la jornada lectiva y el horario general de los centros que imparten la ESO se adecúa a los requisitos aprobados por el consejo escolar del centro, autorizados por la Administración educativa correspondiente, debiendo permitir la realización de todas las actividades académicas y complementarias programadas. Puede ser distinta para las diferentes enseñanzas que se imparten, facilitando no solo cierta optatividad en las asignaturas que cursen los alumnos, sino también un mayor rendimiento de éstos en función de la edad, o un mejor aprovechamiento de los espacios y recursos del centro. En este sentido, son las corporaciones municipales las que, junto con las Asociaciones de Padres y Madres de Alumnos (AMPAs), podrán hacer uso fuera del horario lectivo de los equipamientos disponibles en cada centro, para realizar actividades educativas, culturales, deportivas, u otras de carácter social. 


\section{El ocio como tiempo de uno mismo: la mirada de los adolescentes}

Las realidades sociales y las condiciones de vida en las que los adolescentes proyectan su cotidianeidad, determinan -en parte- el valor que le otorgan al tiempo libre y a las prácticas de ocio en las que participan habitualmente, siendo aquellas sustancialmente diferentes en función del medio -rural o urbano- del que proceden. No sólo por lo que hacen en ellas, sino y fundamentalmente, por las posibilidades o limitaciones que ofrecen sus respectivos contextos en el acceso, o no, a unos determinados recursos, iniciativas o actividades. En este sentido, debemos significar que del total del alumnado de ESO encuestado, un $82,6 \%$ reside en núcleos urbanos o en las afueras de una ciudad; esta circunstancia incide significativamente en la cantidad y diversidad de las actividades en las que participan, ya que suelen contar con una mayor y más variada oferta de equipamientos y programas socioculturales, recreativos y deportivos.

En todo caso, y partiendo de estas premisas, cuando se analiza comparativamente el tiempo que el alumnado de la ESO dedica a determinadas actividades de ocio, se aprecian cambios importantes en relación con las que solían practicar en la Educación Primaria (según datos obtenidos en un estudio previo), con un porcentaje elevado de niños/as que ocupaban buena parte de su tiempo libre jugando con videoconsolas y viendo la televisión. Ahora, cursando la Educación Secundaria Obligatoria, las actividades que ocupan sus "tiempos libres" son fundamentalmente académicas: estudiar y hacer los deberes son las tareas a las que dicen dedicar más tiempo fuera el horario escolar, llegando en un $46,5 \%$ de los casos a destinarles entre 1 y 3 horas diarias. Muchas de estas actividades, que suponen un continuum del tiempo escolarizado (BRAGA, 2007), normalmente se llevan a cabo en el domicilio familiar o en centros específicos de enseñanza a los que asisten -en su tiempo extraescolar-para incrementar su capital académico, garantizar un mejor rendimiento o compensar las carencias formativas de la escuela. Su inicial caracterización como un tiempo de libre disposición por parte de los alumnos, deviene en un tiempo intensamente ocupado, a menudo condicionado por los "deberes" que imponen los profesores, los resultados académicos (bajas calificaciones) o el afán de los padres-madres en procurar una mejor formación para sus hijos/as, ampliándola al dominio de un idioma, al desarrollo de una afición artística, o a la adquisición de unas determinadas competencias o habilidades.

Como puede constarse en la tabla $\mathrm{n}^{\circ} 1$, además del "estudio y los deberes escolares", un porcentaje elevado de respuestas corresponden a actividades 
como "navegar por internet", "chatear y participar en redes sociales". En este sentido, considerando que los niveles de conexión a internet son altos, el uso de las tecnologías de la información y la comunicación ocupan una parte significativa del "tiempo libre" de los adolescentes, la mayoría dentro del hogar, aunque a menudo utilizándolas como un recurso para hacer trabajos y resolver las tareas escolares. Al margen de las finalidades asociadas a su uso, todo indica que los adolescentes dedican una parte importante de su vida cotidiana a las pantallas, coincidiendo con su habitual caracterización como una generación digitalizada, que construye sus señas de identidad a través de diferentes sistemas de comunicación (CALLEJA; GUTIÉRREZ, 2012).

De las actividades realizadas a diario por el alumnado de la ESO, llama la atención que "leer" o "escuchar música" hayan experimentado un cambio importante en los últimos años. Por los datos obtenidos, cabría pensar que constituyen actividades marginales en el ocio adolescente, puesto que un $88,2 \%$ afirma que lee menos de una hora diaria y un $75,5 \%$ que dedica el mismo tiempo a escuchar música; un dato que contrasta con el que ofrece el Barómetro de hábitos de lectura (MINISTERIO DE CULTURA, 2011) cuando indica que un 58\% de la población española mayor de 14 años lee en su tiempo libre. Lo que nos lleva a pensar, tal y como se argumenta en el mismo Informe, que el desarrollo de los medios tecnológicos y audiovisuales, la generalización de nuevos soportes técnicos, unidos a la diversidad de modos de acercarse a este tipo de actividades, han variado notablemente en los hábitos de los adolescentes, ya que un elevado porcentaje de los encuestados (exactamente el $52,5 \%$ ) afirma que lee a través de soportes digitales. Lo mismo sucede cuando señalan como actividad "escuchar música", en cuya práctica, y a medida que aumenta la edad, la población más joven pasa de ser un sector de referencia para la industria sonora a consumidores esporádicos; una circunstancia que obliga a diversificar la oferta y los sistemas de acceso, ya que muchos adolescentes y jóvenes prefieren descargarla de internet o emplear el ordenador para poder escucharla. 
CABALLO, M. B.; CARIDE, A.J.; GRADAÍLLE, R. Entre los tiempos escolares y los tiempos de ocio...

TABLA 1 - TIEMPO QUE DEDICA EL ALUMNADO DE LA ESO A DISTINTAS ACTIVIDADES REALIZADAS EN LOS DÍAS LECTIVOS

\begin{tabular}{|l|c|c|c|c|c|}
\hline \multirow{2}{*}{ Tipo de actividad realizada } & \multicolumn{5}{|c|}{ Tiempo dedicado } \\
\cline { 2 - 6 } & $\begin{array}{c}\text { nada - 15 } \\
\text { minutos }\end{array}$ & $\begin{array}{c}\text { de 15 a 60 } \\
\text { minutos }\end{array}$ & $\begin{array}{c}\text { de 1 a 3 } \\
\text { horas }\end{array}$ & $\begin{array}{c}\text { más de 3 } \\
\text { horas }\end{array}$ & $\begin{array}{c}\text { no saben no } \\
\text { contestan }\end{array}$ \\
\hline Navegar por internet & 27,7 & 38,9 & 24,1 & 7,8 & 2,0 \\
\hline $\begin{array}{l}\text { Chatear-participar en redes } \\
\text { sociales }\end{array}$ & 31,7 & 33,6 & 24,7 & 8,8 & 1,2 \\
\hline Estudiar, hacer los beberes & 7,5 & 36,0 & 46,5 & 8,6 & 1,5 \\
\hline $\begin{array}{l}\text { Ayudar en las tareas } \\
\text { domésticas }\end{array}$ & 36,7 & 49,8 & 10,1 & 1,7 & 1,8 \\
\hline Estar com la familia & 11,4 & 30,0 & 26,5 & 30,0 & 1,9 \\
\hline Estar sin hacer nada & 69,7 & 19,1 & 6,1 & 3,2 & 1,9 \\
\hline Ver la relevisión & 13,2 & 45,1 & 32,9 & 8,1 & 0,9 \\
\hline
\end{tabular}

(Estimación a partir de los ítems que obtienen mayores porcentajes de respuestas en el alumnado, $\mathrm{N}=3306$ )

Fuente: Caride (Dir.) y otros (2011): Tiempos escolares y tiempos de ocio: problemáticas especificas y alternativas pedagógico-sociales en la vida cotidiana del alumnado de Educación Secundaria Obligatoria (ESO): cuestionario aplicado al alumnado en el marco del Proyecto de Investigación PSI2008-04308PSIC.

Por otra parte, entre las actividades predominantes en el uso de su tiempo libre, los adolescentes sitúan el hecho de "compartir momentos familiares", ya que -según expresa un $30 \%$ de los que dieron respuesta al cuestionario- le dedican más de 3 horas diarias; un dato que concuerda con la percepción que tienen respecto al tiempo que pasan en familia, considerándolo como "mucho-bastante" en un 52,2\% de los casos, si bien transcurren -como mínimo durante una hora diaria- frente al televisor $(45,1 \%)$. Además, el hecho de disponer de tiempo libre mientras permanecen en el hogar, también parece posibilitar una colaboración más efectiva en las tareas domésticas (49,8\%), comenzando a asumir en estas edades las responsabilidades que requiere la convivencia familiar.

La tipología de actividades que realizan, así como la frecuencia con que lo hacen -ya sea en días lectivos o durante los fines de semana-, acentúa el destacado papel que tienen las actividades de ocio en "compañía de los amigos" $(93,0 \%)$ y la participación en alguna práctica deportiva (80,5\%). Las que implican relacionarse socialmente en espacios abiertos, públicos y/o urbanos son los elegidos preferentemente por este colectivo, siendo una práctica habitual "estar en la calle o en el parque" (81,2\%), "ir de compras" $(83,2 \%)$, "caminar y pasear" $(74,7 \%)$. 
En todo caso, no puede obviarse que las actividades con un carácter más marcadamente educativo y/o curricular, como asistir a "clases particulares", no superan el $42 \%$ del total; ni que las identificadas como extraescolares (clases de música, pintura...), apenas representan el 32 por ciento del total de encuestados. Resulta llamativo que, a diferencia de las percepciones que suelen hacerse sobre la participación de los adolescentes en "botellones" (consumo colectivo de bebidas alcohólicas), un 64,2\% afirma no asistir nunca a ellos. Una tendencia que se mantiene a lo largo de la semana y durante el fin de semana. A lo largo de la semana acostumbran a reunirse un par de días con sus amigos fuera del centro escolar (30,2\%), mientras que las clases de apoyo curricular son -en uno o dos días a la semana- una de las actividades que agrupa más adolescentes $(23,5 \%)$, aún cuando son las prácticas deportivas las que presentan una regularidad más visible, al afirmar un $24,7 \%$ que las realiza 3 o 4 días a la semana. Analizando sus preferencias durante el fin de semana, los sábados son los días destinados a "salir por la noche" (31,2\%) y a "ir de compras" (22,4\%). Más allá de estas prácticas "quedar con los amigos" (38,7\%) es la actividad más relevante del fin de semana, que tiene su prolongación en el hecho de estar en la calle con ellos $(28,6 \%)$ o de jugar en espacios urbanos abiertos $(15,5 \%)$.

\section{El ocio como tiempo de los otros (adolescentes): la mirada del profesorado}

Conocer las lecturas que hace el profesorado de ESO acerca de los tiempos libres de los adolescentes, sobre sus realidades y potencialidades, posibilita transferir una parte de sus percepciones a la responsabilidad que le atribuyen -o no- a los centros escolares en la Educación del Ocio de este colectivo. Los datos que se desprenden del cuestionario aplicado al profesorado dibujan un retrato, en general negativo, de cómo ocupan los tiempos cotidianos sus alumnos en los días lectivos, considerando que dedican mucho o bastante tiempo a chatear y comunicarse por las redes sociales, a jugar con videoconsolas y en el ordenador o a navegar por internet; en contraposición, consideran que no dedican tiempo, o apenas una parte de él, a leer, a pasear o a colaborar en las tareas domésticas. A lo que se añade el énfasis que ponen en el poco tiempo que se dedican a estudiar y a hacer los deberes escolares.

El profesorado considera que sus alumnos -adolescentes-ocupan su vida fuera del centro escolar en actividades que están muy mediatizadas por la tecnología, ya sea para entretenerse y divertirse, o para comunicarse en el espacio 
virtual. Una realidad muy distante a la de los centros de Educación Secundaria, con una disponibilidad muy limitada en el uso de los medios tecnológicos, poniendo de manifiesto la brecha existente entre las dinámicas sociales y las escolares. Cabe señalar, además, que esta percepción acerca de la ocupación del tiempo de los adolescentes (sus alumnos) no coincide con la que tienen los adolescentes de sí mismos (ver tabla $\mathrm{n}^{\mathrm{o}} 1$ ).

Los datos que figuran en la tabla $\mathrm{n}^{\circ} 2$ son un extracto del conjunto de respuestas del profesorado al cuestionario que les fue aplicado, demasiado extensas para ser incorporadas a la argumentación que presentamos. En ellas, haciendo uso de una escala tipo Likert (1-5), llama la atención el elevado porcentaje de respuestas concentrado en el punto medio, sobre todo en los ítems que aluden al trabajo académico o peri-extra-académico.

TABLA 2- TIEMPO QUE DEDICA EL ALUMNADO DE LA ESO A DISTINTAS ACTIVIDADES REALIZADAS EN LOS DÍAS LECTIVOS

\begin{tabular}{|l|c|l|c|}
\hline Dedican bastante o mucho tiempo a ... & $\%$ & Dedican nada o poco tiempo a.. & $\%$ \\
\hline Chatear y redes sociales & 84.4 & Leer & 79.7 \\
Jugar con videoconsolas/ordenador & 84.0 & Caminar/pasear & 75.0 \\
Navegar por internet & 83.9 & Ayudar en tareas domésticas & 62.5 \\
Ver la televisión & 76.8 & Estar en bares/pubs & 54.2 \\
Usar el móvil & 72.1 & Ir al ciber/salón de juegos & 45.9 \\
Estar con amigos/as & 54.6 & Estudiar/hacer deberes & 42.3 \\
Escuchar música & 50.4 & Ir al botellón & 42.0 \\
& & Estar con la familia & 41.5 \\
\hline
\end{tabular}

(Estimación a partir de los ítems que obtienen mayores porcentajes de respuestas en el profesorado, $\mathrm{N}=480$ )

Fuente: Caride (Dir.) y otros (2011): Tiempos escolares y tiempos de ocio: problemáticas especificas y alternativas pedagógico-sociales en la vida cotidiana del alumnado de Educación Secundaria Obligatoria (ESO): cuestionario aplicado al profesorado en el marco del Proyecto de Investigación PSI2008-04308PSIC.

En un rango que oscila entre el $40,9 \%$ y el $51,4 \%$, el profesorado estima que su alumnado dedica "algo" de tiempo a practicar deporte, a realizar actividades extraescolares fuera del centro, a estudiar y a hacer los deberes, o acudir a clases particulares. Este hecho, unido al elevado porcentaje de los profesores que "no saben/no contestan" (entre un 10,2\% y un 19,2\%) cuando se les pregunta acerca de los tiempos que invierten sus alumnos en "estar con la familia", "ayudar en casa", "ir de botellón" o "de compras", revelan, a nuestro entender, un conocimiento insuficiente del entorno en el que se mueven, así como de lo que es su vida cotidiana más allá del horario lectivo. Si circunscribimos esos tiempos 
a los "tiempos libres", el profesorado manifiesta de forma mayoritaria que sus alumnas y alumnos no los utilizan correctamente, ya que ni saben aprovecharlos ni distribuirlos. Sigue prevaleciendo la opinión de que hacen un uso excesivo de las tecnologías, derivando en un ocio muy condicionado por los entornos virtuales, que además de propiciar el sedentarismo, merma las posibilidades para unas relaciones personales más directas e intensas.

En opinión del profesorado, el grupo de adolescentes que gestiona y aprovecha bien los tiempos que tiene de libre disposición (su tiempo), es reducido. Además, cuando se pretende profundizar en la identificación de los problemas que tienen los colectivos adolescentes en sus tiempos libres, se produce, nuevamente, una elevada concentración de profesorado que "no sabe o no contesta"; lo que refuerza nuestra hipótesis del escaso conocimiento que una parte del profesorado tiene sobre realidad cotidiana del alumnado con el que trabaja.

TABLA 3 - PRINCIPALES PROBLEMAS DE LOS ADOLESCENTES EN SU TIEMPO LIBRE

\begin{tabular}{|l|c|c|c|}
\hline Problemas identificados & $\begin{array}{c}\text { no tienen } \\
\text { ningún } \\
\text { problema }\end{array}$ & $\begin{array}{c}\text { sí tienen algún } \\
\text { problema }\end{array}$ & $\begin{array}{c}\text { no sabe } \\
\text { no contesta }\end{array}$ \\
\hline $\begin{array}{l}\text { Disponibilidad de tiempo } \\
\text { personal de ocio }\end{array}$ & 41.3 & 32.5 & 23.5 \\
\hline Oferta de iniciativas & 27.5 & 38.3 & 34.2 \\
\hline Infraestructuras y recursos & 34.4 & 34.0 & 31.6 \\
\hline Aspectos económicos & 27.3 & 31.7 & 41.0 \\
\hline Motivaciones personales & 12.7 & 43.5 & 43.8 \\
\hline
\end{tabular}

Perspectiva del profesorado $(\mathrm{N}=480)$.

Fuente: Caride (Dir.) y otros (2011): Tiempos escolares y tiempos de ocio: problemáticas especificas y alternativas pedagógico-sociales en la vida cotidiana del alumnado de Educación Secundaria Obligatoria (ESO): cuestionario aplicado al profesorado en el marco del Proyecto de Investigación PSI2008-04308PSIC.

Dejando al margen el porcentaje de quienes "no saben o no contestan" (que oscila entre el 23,5 y el 43,8 por ciento de las "no respuestas"), la mayor parte del profesorado identifica como principal problema del tiempo libre de los adolescentes la escasa motivación que tienen en todos los campos: los estudios, el ocio y la vida, en general. En las preguntas abiertas del cuestionario, que permiten matizar estas afirmaciones, buena parte del profesorado se refiere a la desmotivación y al aburrimiento de los adolescentes, así como a la falta de valoración de todo aquello que suponga esfuerzo. Algunas respuestas apuntan hacia la falta de estímulo en el ámbito familiar, junto con la ausencia de criterios, por parte de los adolescentes, para decidir qué hacer, a lo que se une la 
influencia que ejercen determinados modelos sociales y el grupo de sus iguales en los posicionamientos que mantienen.

En la valoración que hace el profesorado sobre el tiempo personal (de ocio) del que disponen sus alumnos, predominan las respuestas que apuntan hacia la sobrecarga de actividades extraescolares: un aspecto en el que inciden investigaciones como la realizada recientemente en esta misma franja de edad en la provincia de Salamanca (MUÑOZ et al., 2011, p. 147-148), poniendo de manifiesto como el exceso de actividades dirigidas o impuestas anula las posibilidades de una vivencia satisfactoria del ocio: "la falta de libertad de los chicos a la hora de elegir, el deseo de los padres de que hagan los hijos lo que ellos no pudieron hacer, la sobresaturación de actividades -deportes, música, idiomas, etc.-, hacen perder el objetivo con el que fueron elegidas esas actividades para ocupar el tiempo libre".

Frente a estos argumentos, que se concentran en el ocio adolescente urbano, surgen los que denuncian la escasez de oferta en el medio rural y la problemática que supone la dispersión de la población para configurar grupos de iguales; la inadecuación de los horarios de las actividades y las dificultades de transporte, la dependencia de las tecnologías para llenar los tiempos libres, la soledad en la que se viven estos tiempos, o las dificultades que los adolescentes tienen para organizarlos, unida a la falta de supervisión de lo que hacen por parte de sus familias. El desconocimiento de la oferta existente, así como la escasez de iniciativas específicas dirigidas al colectivo adolescente son algunas de las cuestiones en las que inciden, mostrando una elevada confluencia con otros estudios, como el realizado en la ciudad catalana de Lleida (BASELLS et al., 2011, p. 75-77) en el que, tras analizar todos los recursos disponibles para la infancia y la adolescencia en los ámbitos de la cultura y el tiempo libre, se concluye que, efectivamente, hay una oferta reducida para esta franja de edad, así como problemas con la difusión de información sobre los espacios y actividades de ocio que se promueven desde la iniciativa pública y privada.

El profesorado que ha dado respuesta al cuestionario insiste en que los adolescentes no tienen criterios a la hora de decantarse por unas u otras actividades; "una cosa es tener tiempo y otra saberlo usar adecuadamente [...]. Ello obliga a las familias y a los colectivos e instituciones respectivas, escolares y sociales, a llevar a cabo programas cuyo objetivo sea ayudar a los chicos a ordenar y distribuir mejor su tiempo" (MUÑOZ, 2011, p. 149-150). En esta misma dirección apuntaba, ya en 1994, la Carta Internacional de Educación del Ocio promovida por la Asociación Mundial de Ocio y Recreación (WLRA), al enfatizar la responsabilidad de los centros educativos, de las familias y comunidades en visibilizar la importancia que tienen los tiempos libres en el desarrollo humano, así como en la necesidad de educar para transformarlos en experiencias de ocio. 
En la investigación que presentamos, de 316 respuestas formuladas acerca de esta cuestión, sólo cinco aluden al centro escolar como un agente que puede -y debe-intervenir en los tiempos de ocio: la primera, refiere los problemas que derivan de la ausencia de transporte escolar en horario de tarde, afirmando que " $s i$ hubiera autobús, el centro podría ser un punto de ocio saludable"; la segunda, destaca las tutorías como un recurso del que podrán hacer uso los profesores para trabajar aspectos relacionados con los tiempos libres de los adolescentes; la tercera, también en esta dirección, propone que se podrían "planificar con las familias en las tutorías unos horarios sensatos"; la cuarta, asume que desde los centros se debería reducir la carga de trabajo escolar para casa, de modo que el alumnado pueda disponer de más tiempo libre de gestión autónoma; y, por último, la de un profesor que destaca el papel que debería asumir el Departamento de Orientación Escolar del Instituto para dar a conocer las alternativas de ocio que se ofrecen, tanto desde el centro educativo como desde otras entidades y colectivos de la zona. Sólo dos respuestas consideran que ha de implicarse a los adolescentes, ya "nunca se cuenta con ellos para ver qué necesitan o cuáles son sus preferencias"; y de que hay un "insuficiente número de educadores y gestores sociales", en alusión a la conveniencia de incorporar otros perfiles que trabajen temas de educación del ocio y dinamización de recursos comunitarios. Y todo ello cuando la libertad de elección y la autonomía son dos elementos intrínsecos a la definición del ocio (CUENCA, 2011).

En lo que atañe a la disponibilidad de infraestructuras y recursos, se repiten las líneas argumentales de respuestas anteriores, insistiendo en las diferencias que se dan entre contextos rurales y urbanos, y en la necesidad de incrementar la oferta pública en el ámbito de la cultura, el deporte y las relaciones sociales, especialmente por parte de las Administraciones Locales. Sin duda, por su proximidad a la ciudadanía y su potencial de trabajo en red con otras entidades y recursos de la comunidad, están llamadas a asumir un protagonismo en el liderazgo de proyectos educativos territoriales, en línea con la filosofía de las “ciudades educadoras" (CABALLO; GRADAÍLLE, 2008). En lo que respecta a los aspectos económicos, la mayoría de las respuestas aluden a las dificultades de buena parte de las familias de su alumnado (situaciones de paro, precariedad laboral, pérdida de poder adquisitivo...), que afectan su acceso al ocio; aunque también hay bastantes opiniones que inciden en que los adolescentes manejan demasiado dinero para la edad que tienen, así como - de nuevo- a la falta de criterio para un uso adecuado del mismo. Son escasas las respuestas que expresan opiniones complementarias, aún cuando destacan las reflexiones que acentúan el contraste que existe entre la desprotección de muchos adolescentes por parte de sus familias y la sobreprotección de otros, aludiendo a que "en demasiados hogares no hay orden ni control. Los adultos del hogar son adolescentes, igual 
que sus hijos". Sólo un docente introduce la cuestión del ocio de consumo frente a un ocio más creativo, como un problema que también afecta al colectivo adolescente, afirmando que "es más fácil consumir ocio que pensar en cómo divertirse alejados del ocio de obligado consumo".

\section{Educar el ocio adolescente, una tarea pendiente}

Por lo que hemos expuesto, la ausencia de una Educación en, para y del Ocio subyace a buena parte de las respuestas dadas: los adolescentes no saben cómo gestionar sus tiempos ni cómo aprovechar los tiempos libres, están desmotivados, predominan los usos vinculados a las tecnologías obviando otros recursos y espacios, etc.; un conjunto de afirmaciones que contrastan con las respuestas de un $34 \%$ del profesorado que afirma que los centros educativos no tienen ninguna responsabilidad - o muy escasa- en la educación del ocio de este colectivo; en definitiva, de sus propios alumnos. Que para un $37,9 \%$ se esté educando "algo" para el ocio y que para un $47,1 \%$ no se asuma en "nada" o "poco" esta tarea, pone de relieve la baja preocupación que esta temática suscita entre el profesorado; más aún, cuando un elevado porcentaje de los docentes -cercano al 75 por ciento de los encuestados- no consideran que deban abrirse las instalaciones escolares los fines de semana o en las vacaciones. Ambas opiniones contrastan con la aceptación mayoritaria de la necesidad de incorporar a otros profesionales de la educación en los centros educativos para desarrollar programas o iniciativas relacionadas con el ocio: un 64,6\% están bastante o muy de acuerdo con ello.

No obstante, si los tiempos de apertura y cierre de los centros educativos no se hacen más flexibles, si no se integran en la vida comunitaria como un recurso socioeducativo más, ¿en qué tiempos y cómo educarán las escuelas y los institutos para el ocio? ¿Cómo y en qué tiempos se incorporarán otros profesionales de la educación para desarrollar estas tareas? Sin duda, la educación del ocio, muy especialmente en la adolescencia, es una responsabilidad que han de compartir diferentes agentes; entre ellos, los centros educativos, que deben asumir una función que vaya mucho más allá de los procesos de enseñanza-aprendizaje curriculares o la formación que ocupa los horarios lectivos, para convertirse en espacios abiertos a la convivencia y la acción sociocomunitaria: "un referente lúdico intergeneracional, equipamiento de proximidad para el encuentro, la práctica físico-deportiva saludable, la creatividad y el incremento del bienestar social" (FRAGUELA; POSE; VARELA, 2011, p. 65). Unos centros educativos 
que, en convergencia con las familias, las Administraciones públicas, el tejido asociativo y la iniciativa empresarial de cada territorio, hagan posible que los y las adolescentes vivencien su formación de un modo más integral, también en los tiempos libres y en las alternativas que de ellos podrán derivarse para un ocio más satisfactorio, por los valores que promueve y por las contribuciones que de sus prácticas podrán derivarse para mejorar su bienestar individual y colectivo.

\section{REFERENCIAS}

BASELLS, M. A. et al. La infància i l'adolescència de la ciutat de Lleida. Informe 2009-2010. Lleida: Universitat de Lleida, 2011.

BRAGA, G. M. (Coord.). La situación de la infancia y la adolescencia en Asturias. Oviedo: Principado de Asturias, 2007.

CABALLO, M. B.; CARIDE, J. A. (Coords.). Monográfico "Tiempos educativos y sociales". Educación Social. Revista de Intervención Socioeducativa, n. 47, p. 11-94, 2011.

CABALLO, M. B.; GRADAÍLLE, R. La educación social como práctica mediadora en las relaciones escuela-comunidad local. Pedagogía Social. Revista Interuniversitaria, n. 15, tercera época, p. 45-55, 2008.

CALLEJO, J.; GUTIÉRREZ, J. (Coords.). Adolescencia entre pantallas: identidades juveniles en el sistema de comunicación. Barcelona: Gedisa, 2012.

CARBONELL, J. Una educación para mañana. Barcelona: Octaedro, 2008.

CASTELLS, M. La era de la información. Economía, sociedad y cultura: la sociedad red (vol. I). Madrid: Alianza Editorial, 1997.

CAVET, A. Rythmes scolaires: pour une dynamique nouvelle des temps éducatifs, Dossier d'actualité de la VST, n. 60. Disponible en: <http://www.inrp.fr/vst/ LettreVST/60-fevrier-2011.php>. Acceso en: 08/01/2012.

CEBRIÁN, J. L. La red: cómo cambiarán nuestras vidas los nuevos medios de comunicación. Barcelona: Círculo de Lectores, 1998.

COMPÈRE, M.-M. (Ed.). Historie du temps scolaire en Europe. París: INRP, 1997.

CUENCA, M. Pedagogía del Ocio: modelos y propuestas. Bilbao: Universidad de Deusto, 2004. 
CUENCA, M. La fuerza transformadora del ocio. In: CUENCA, M.; AGUILAR, E.; ORTEGA, C. (Coords.). Ocio para innovar. Documentos de Estudios de Ocio, n. 41. Bilbao: Universidad de Deusto, 2011, p. 17-82.

DELORS, J. (Dir.). La educación encierra un tesoro: informe a la UNESCO de la Comisión Internacional sobre la Educación para el siglo XXI. Madrid: SantillanaUNESCO, 1996.

DURÁN, M. A. El valor del tiempo: ¿cuántas horas te faltan al día? Madrid: EspasaCalpe, 2007.

DURÁN, M. A.; ROGERO, J. La investigación sobre el uso del tiempo. Cuadernos metodológicos, n. 44. Madrid: Centro de Investigaciones Sociológicas, 2010.

ELIZALDE, R. Resignificación del ocio: aportes para un aprendizaje transformacional. Polis: Revista Académica de la Universidad Bolivariana, n. 25, 2010. Disponible en: $<$ http://www.revistapolis.cl/25/art25.htm>. Acceso en: 20/11/2011.

ESCOLANO, A. Tiempos y espacios para la escuela: ensayos históricos. Madrid: Biblioteca Nueva, 2000.

FOUCAULT, M. Vigilar y castigar. Madrid: Siglo XXI, 1982.

FRAGUELA, R.; POSE, H.; VARELA, L. Nuevos tiempos para la acción socioeducativa municipal: deporte, educación y cultura. Educación Social. Revista de Intervención Socioeducativa, n. 47, p. 63-73, 2011.

GIMENO, J. El valor del tiempo en educación. Madrid: Morata, 2007.

HARGREAVES, A. El tiempo y el espacio en el trabajo del profesor. Revista de Educación, Madrid, n. 298, p. 31-53, 1992.

HUSTI, A. Del tiempo escolar uniforme a la planificación móvil del tiempo. Revista de Educación, Madrid, n. 298, p. 271-305, 1992.

MARRAMAO, G. Kairós. Apología del tiempo oportuno. Barcelona: Gedisa, 2008.

MINISTERIO DE CULTURA: Informe de resultados. Hábitos de lectura y compra de libros en España 2011. Disponible en: <http://www.mcu.es/libro/docs/MC/ Observatorio/pdf/HLCLE_semestre1_2011.pdf>. Acceso en: 10/01/12.

MUÑOZ, J. M. (Ed.). Adolescencia y tiempo libre. Análisis y propuestas educativas en Salamanca. Salamanca: Ediciones Universidad de Salamanca, 2011.

PEREYRA, M. A. En el comienzo de una nueva época. Cuadernos de Pedagogía, n. 349, p. 53-59, 2005. 
CABALLO, M. B.; CARIDE, J.A.; GRADAÍLLE, R. Entre los tiempos escolares y los tiempos de ocio...

ROMERO, C. Conocimiento del tiempo educativo. Barcelona: Laertes, 2000.

TABBONI, S. Les temps sociaux. París: Armand Colin, 2006.

WLRA. International Charter for Leisure Education, ELRA (European Leisure and Recreation Association), verano, p. 13-16, 1994; en español: CUENCA, M. Pedagogía del ocio: modelos y propuestas. Bilbao: Universidad de Deusto, p. 315-324, 2004.

Texto recebido em 23 de março de 2012.

Texto aprovado em 15 de abril de 2012. 
